\title{
DETECÇÃO DO ANALGÉSICO PARACETAMOL NO CÓRREGO DA ONÇA, TRÊS LAGOAS - MS
}

\author{
Juliana Heloisa Pinê Américo ${ }^{1}$
}

Alessandro Minillo ${ }^{2}$

Sérgio Luís de Carvalho ${ }^{3}$

RESUMO: A contaminação dos ecossistemas aquáticos por fármacos intensificou-se nos últimos anos devido ao aumento da produção e ao consumo desenfreado destas substâncias pela população. Estes compostos são encontrados em águas naturais e em efluentes de Estações de Tratamento de Esgoto (ETE), comprovando que sua presença em aguas naturais é um indicativo de contaminação por águas residuárias de ETE. $O$ presente trabalho teve como objetivo diagnosticar a presença e determinar a concentração do analgésico paracetamol em seis pontos de amostragem do Córrego da Onça, município de Três Lagoas, Mato Grosso do Sul. Assim, foram coletadas mensalmente, em um período de 12 meses amostras de água (1L) em seis pontos distintos representando diferentes seções do córrego. As amostras foram submetidas ao processo de extração em fase sólida e analisadas por cromatografia líquida de alta eficiência. O paracetamol foi detectado nos seis pontos de coleta, sendo mais frequente no ponto localizado a jusante da ETE do município. Os resultados evidenciam a contaminação no córrego por paracetamol, possivelmente devido aos indícios de ligações clandestinas de esgoto sanitário e/ou não eficiência do sistema utilizado pela ETE do município de Três Lagoas - MS.

Palavras-chave: Fármaco. ETE. Ambiente aquático.

\footnotetext{
${ }^{1}$ Doutoranda em Aquicultura, Centro de Aquicultura da UNESP. E-mail: americo.ju@gmail.com

${ }^{2}$ Docente, Universidade Estadual do Mato Grosso do Sul (UEMS).E-mail: alminillo@yahoo.com.br

${ }^{3}$ Docente, Faculdade de Engenharia de Ilha Solteira (UNESP). E-mail: sergicar@bio.feis.unesp.br
} 


\section{INTRODUÇÃO}

A crescente expansão dos grandes centros urbanos e industriais tem promovido uma degradação progressiva da qualidade das águas superficiais devido ao aumento da contaminação de rios, lagos e reservatórios por compostos xenobióticos. No Brasil, principalmente nas regiões mais populosas, esta situação é altamente previsível, considerando seu crescimento populacional, econômico e industrial.

Dentre os compostos xenobióticos cada vez mais presentes nos ambientes aquáticos, os fármacos vêm recebendo maior atenção, em razão de sua capacidade de persistência no meio ambiente (GHISELLI e JARDIM, 2007). A ocorrência de fármacos residuais no esgoto doméstico e águas naturais é um importante tópico internacional. Pesquisas demonstram que estes fármacos e seus metabolitos estão presentes em ambientes aquáticos em várias partes do mundo (BILA e DEZOTTI, 2003), e como as Estações de Tratamento de Esgoto (ETE) não são eficientes na remoção destes resíduos este tipo de contaminação torna-se mais preocupante já que estes efluentes são lançados diretamente em rios provocando a contaminação das águas superficiais (BAUTITZ, 2006).

A presença de fármacos residuais no meio ambiente pode causar efeitos adversos em organismos aquáticos e terrestres, além de prejuízos a saúde humana. No entanto, ainda pouco se sabe sobre o destino e o comportamento destas substâncias no ambiente aquático, assim como não está claro quais organismos são afetados e em que grau.

O objetivo do presente trabalho foi diagnosticar a presença e determinar a concentração do analgésico paracetamol em seis pontos de amostragem do Córrego da Onça, município de Três Lagoas, Mato Grosso do Sul durantes doze meses consecutivos no período de outubro de 2008 a setembro de 2009, já que é um fármaco muito utilizado em regiões endêmicas da dengue. 


\section{MATERIAL E MÉTODOS}

\subsection{Caracterização da área de estudo}

O município de Três Lagoas está localizado na porção leste do Estado do Mato Grosso do Sul com vegetação característica de cerrado e mata atlântica. Possui uma população de 89.493 habitantes e uma área de unidade territorial de 10.206,37 km² (IBGE, 2009).

O Córrego da Onça localizado no município de Três Lagoas - MS está com a qualidade de sua água afetada devido à intensa degradação que sofreu nos últimos anos fruto do inadequado uso e ocupação do solo no seu entorno, além do despejo inapropriado de esgoto sanitário.

Considerando a problemática desse ambiente, realizou-se um plano de monitoramento nesse corpo hídrico que obteve dados de 12 meses consecutivos, no período de outubro de 2008 a setembro de 2009, a fim de se avaliar a degradação causada pela contaminação deste meio pelo fármaco de uso humano paracetamol, amostrando-se seis pontos devidamente georreferenciados, estes denominados: Lagoa do Meio (1), Lagoa Maior (2), Final da Canalização (3), Jusante da Estação de Tratamento de Esgoto (ETE), (4), Novas Nascentes (5) e Foz (6) representando assim diferentes secções e tipos de interferência humana no córrego. A escolha dos pontos de coleta foi determinada de acordo com o uso e ocupação da bacia (Figura 1). 


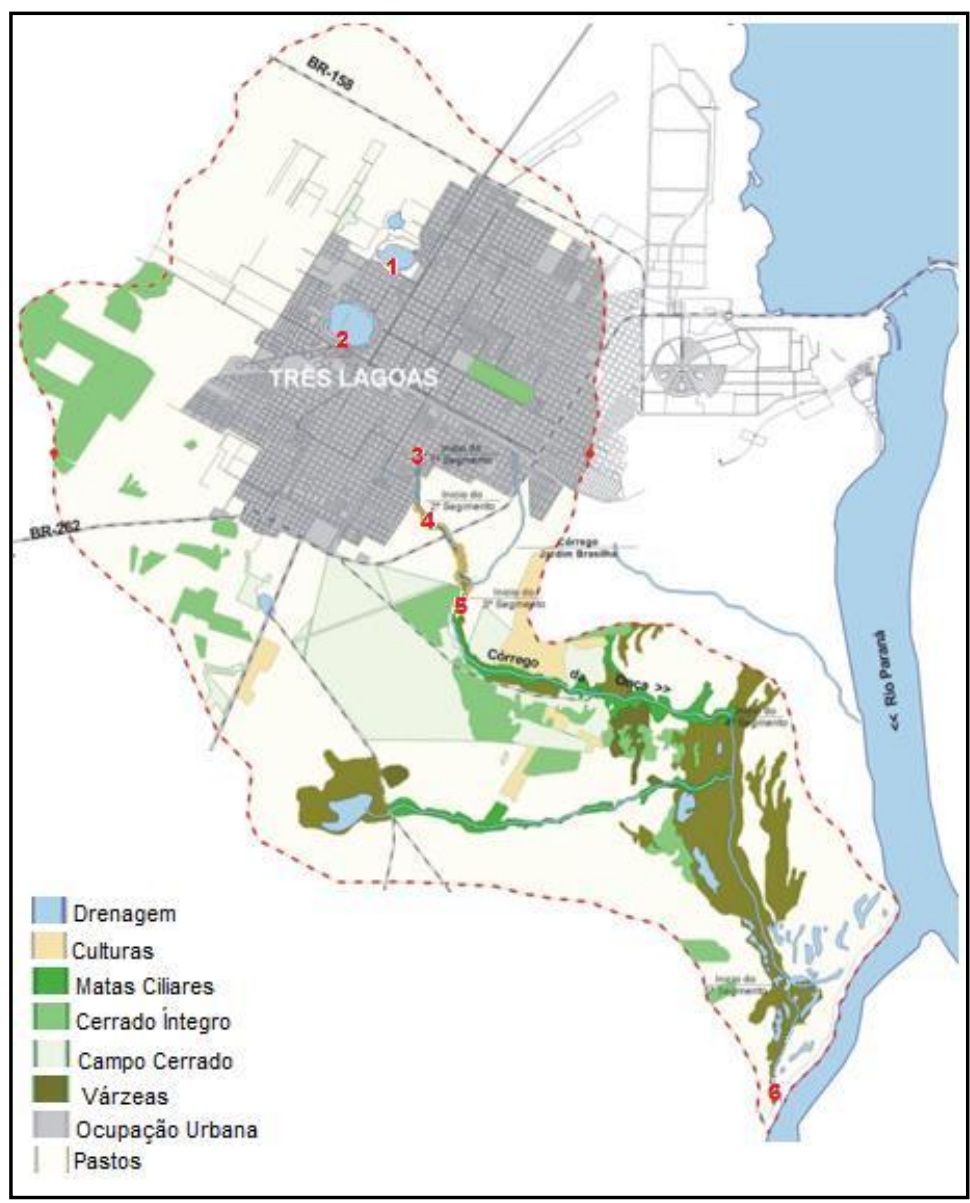

Figura 1. Localização dos seis pontos de coleta no Córrego da Onça, município de Três Lagoas MS. Fonte: Moreira (2006)

\subsection{Fármaco analisado}

O fármaco de uso humano selecionado para ser investigado na água do Córrego da Onça, Três Lagoas - MS foi o paracetamol. A escolha do analgésico deu-se em função da detecção deste composto em outros estudos com análise de amostras ambientais e devido ao seu elevado consumo pela população.

\subsection{Coleta e preparo das amostras de água}




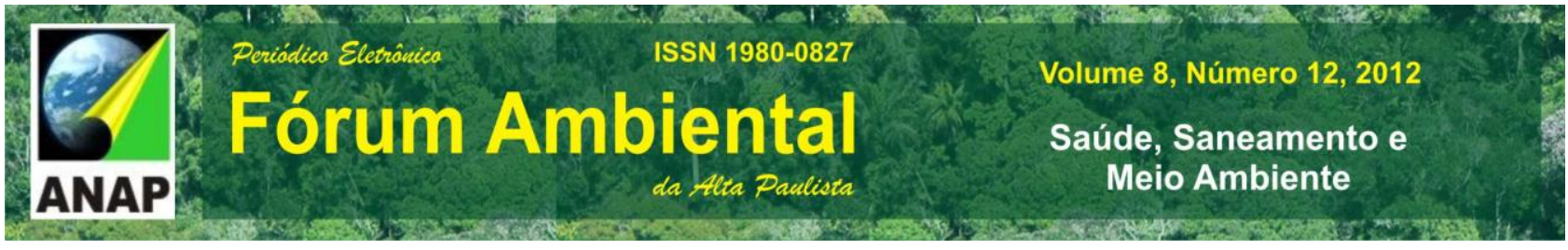

Amostras de água superficial $(1,0$ L) foram coletadas em cada ponto de amostragem com auxilio de baldes graduados e acondicionadas em fracos, sendo transportadas em caixas isotérmicas contendo gelo para posterior análise no Laboratório de Saneamento da Faculdade de Engenharia de Ilha Solteira. Em laboratório as amostras foram filtradas em filtros de fibra de vidro de 0,45 $\mu$ m (Whatman) para a remoção de partículas suspensas, eliminando a ação de possíveis agentes interferentes.

A concentração e extração do fármaco foi realizada segundo o método proposto por Nebot et al. (2007) com adaptações. Para tanto, realizou-se uma pre-ativação das colunas de fase reversa C-18 a base de sílica (AccuBond II ODS-C18 de $500 \mathrm{mg}$ ) com 5,0 $\mathrm{mL}$ de metanol 100\% (J.T.Baker grau HPLC), seguida posteriormente de 5,0 $\mathrm{mL}$ de água Milli - $\mathrm{Q}$ (pH proximo de 3,0). As amostras (1,0 L) foram eluidas nos cartuchos $\mathrm{C} 18$ com velocidade de fluxo de $3,0 \mathrm{~mL} / \mathrm{min}^{-1} \mathrm{com}$ auxilio de bomba a vácuo.

Posteriormente, as colunas de fase reversa C-18 foram lavadas com 5,0 mL de agua Milli-Q (pH próximo de 3,0) e na sequência permaneceram em repouso para secagem a temperatura ambiente por $1 \mathrm{~h}$. Após secagem, o eluato foi recolhido com $2,5 \mathrm{~mL}$ de acetona seguido por $5,0 \mathrm{~mL}$ de metanol $100 \%$. O eluato recolhido foi evaporado a $55^{\circ} \mathrm{C}$ e ressuspendido em $500 \mu \mathrm{L}$ de metanol $100 \%$ para realização das analises cromatográficas.

\subsection{Análise em cromatografia líquida de alta eficiência}

O padrão do paracetamol utilizado na identificação e construção da curva analítica foi obtido pela Eurofarma, o qual apresentou 90\% de grau de pureza. As análises para identificação do analgésico presente nas amostras de água foram efetuadas em um cromatógrafo liquido de alta eficiência (Shimadzu) munido de duas bombas LC-20AT e LC-20AD; Communication Bus Module- CBM-20A (Prominence Communications Bus Module) (Figura 17); Injetor Rheodyne (Rohnert Park, CA, USA) equipado com válvula do tipo loop de $20 \mu \mathrm{L}$. Detector SPD-M20A (Prominence Diode Array Detector) e empregando o software LCsolution. As separações dos picos cromatográficos foram realizadas em uma coluna cromatográfica LC Column Shim-pack C18 (250 mmx 4.6 mm ID, partículas de 5,0 $\mu \mathrm{m})$. 
As fases móveis constituíram-se de metanol (100\%) (fase A) e agua Mili- Q (fase B), ambas acrescidas de 0,1\% de acido trifluoracetico (TFA). O uso de um gradiente linear de 95-50 \% de A por 15 minutos (a temperatura ambiente), com fluxo de 1,0 ml.min.-1 foi utilizado na separação dos picos cromatográficos. O volume de injeção das amostras foi de 25,0 $\mu \mathrm{L}$, sendo as amostras analisadas em triplicata. Os comprimentos de onda utilizados para a detecção dos picos cromatográficos foram de 240; 260 e $280 \mathrm{~nm}$. A identificação do fármaco foi efetuada de acordo com o seu respectivo tempo de retenção e também através do seu perfil espectrofotométrico.

As curvas analíticas foram efetuadas através do método do padrão interno, concomitantemente o limite de detecção (LD) e o limite de quantificação (LQ) (mg. $\mathrm{L}^{-1}$ ) também foram obtidos por meio de planilha de validação proposta por Ribeiro e Ferreira (2008). O LD é definido como a menor quantidade do analito presente em uma amostra que pode ser detectada sob as condições experimentais estabelecidas. Enquanto o LQ é a menor quantidade do analito que pode ser determinada com precisão e exatidão aceitáveis sob condições experimentais estabelecidas (ANVISA, 2003). O limite de quantificação e de detecção do paracetamol é apresentado na Tabela 1.

Tabela 1 - Limite de quantificação (LQ) e detecção (LD) dos fármacos estudados

\begin{tabular}{ccc}
\hline Fármaco & LQ $\left(\mathbf{m g} \cdot \mathbf{~ L}^{-1}\right)$ & LD $\left(\mathbf{m g} \cdot \mathbf{~ L}^{-1}\right)$ \\
\hline Paracetamol & 0,21 & 0,13 \\
\hline
\end{tabular}

Fonte: Ribeiro e Ferreira (2008)

\section{RESULTADOS E DISCUSSÃO}

O paracetamol foi detectado em todos os pontos de amostragem do Córrego da Onça - MS, a presença do fármaco no córrego possivelmente está relacionada ao seu amplo uso pela população da região do estudo, pois é um analgésico muito utilizado em região endêmica de dengue.

Nos pontos localizados na área urbana (P1, P2, P3), o fármaco apresentou-se com menor frequência ( $25 \%$ das amostras), a sua presença nestes pontos pode ser atribuída 
aos possíveis indícios de ligações clandestinas de esgoto doméstico de algumas residências do município que contaminam estes ambientes aquáticos. No P4, 0 analgésico foi detectado em $50 \%$ das amostras coletadas (Tabela 2), esta maior frequência neste ponto de coleta pode ter ocorrido porque o P4 recebe o efluente da ETE do município, e a mesma pode estar concentrando este fármaco ao invés de reduzi-lo, ou seja, o composto presente no efluente é lançado diariamente no córrego que possui vazão quase nula nos períodos de estiagem e, assim, este pode aumentar sua concentração no corpo d'água além da possibilidade de acumular-se no sedimento, uma vez que segundo Halling-Sorensen et al. (1998), 30\% de todos os fármacos desenvolvidos são lipofílicos (hidrosolubilidade < 10\%), sedimentando-se em ambientes aquáticos ou transferindo-se para fase biótica.

Tabela 2 - Concentração de paracetamol, em mg.L-1, no Córrego da Onça - MS no período de Outubro de 2008 a Setembro de 2009

\begin{tabular}{ccccccc}
\hline Meses & \multicolumn{5}{c}{ Pontos Amostrais } & A \\
& P1 & P2 & P3 & P4 & P5 & P6 \\
\hline Out & ND & ND & 2,403 & 0,322 & ND & $*$ \\
Nov & ND & ND & ND & ND & ND & $*$ \\
Dez & ND & ND & 0,600 & 0,455 & ND & 0,701 \\
Jan & ND & ND & ND & 1,514 & 0,282 & 1,877 \\
Fev & 0,190 & ND & ND & 0,317 & 0,282 & ND \\
Mar & ND & ND & ND & ND & ND & D \\
Abr & ND & ND & ND & ND & 0,461 & 0,175 \\
Mai & 0,507 & ND & ND & D & ND & 0,422 \\
Jun & 0,144 & D & ND & ND & ND & ND \\
Jul & ND & 0,192 & D & 0,208 & ND & D \\
Ago & ND & 0,130 & ND & ND & D & 0,398 \\
Set & ND & ND & ND & ND & ND & ND \\
\hline
\end{tabular}

D: Detectado (Quantidade traço do composto) ND: composto não detectado

*: Amostras nao coletadas 
A não detecção do analgésico em alguns meses nos pontos amostrais pode estar associada à biodegradacao do mesmo, visto que segundo Henschel et al. (1997) o paracetamol possui uma biodegradabilidade de 57\%.

As concentrações de paracetamol registradas no Córrego da Onça - MS apresentaram-se maiores em relação a vários estudos com águas superficiais. No Brasil, reportou-se a presença de paracetamol em amostras de água do Ribeirão Anhumas, região metropolitana de Campinas-SP, apenas nos meses de estiagem em concentrações de 0,28 e 13,44 Mg.L-1 (RAIMUNDO, 2007). O fármaco também foi encontrado em águas superficiais (1,2 e 1,9 ng.L-1) que abastecem estações de tratamento de agua (ETAs) na França bem como nos seus respectivos efluentes $(0,8$ e 0,7 ng.L-1) destinados ao consumo humano (VULLIET et al., 2009). Segundo Kasprzyk-Hordern et al. (2008) amostras de diferentes pontos do Rio Taff, no Reino Unido, que recebe efluente de ETE, estavam contaminadas pelo composto $(62,185$ e 388 ng.L-1).

\section{CONCLUSÃO}

Com base nos resultados obtidos é possível concluir que o paracetamol foi detectado nos seis pontos de amostragem do Córrego da Onça - MS. O analgésico foi detectado com maior frequência no ponto localizado à jusante da ETE de Três Lagoas MS, o que era esperado já que os efluentes domésticos são os maiores responsáveis pela contaminação dos ecossistemas aquáticos por fármacos.

A detecção do fármaco em todos os pontos de monitoramento do Córrego da Onça MS é algo preocupante, em razão dos riscos associados à saúde humana e ambiental, seja esta decorrente da água se destinada ao abastecimento da população ou ao comprometimento da biota aquática.

Contaminações hídricas devido a uso de medicamentos veterinários nas propriedades próximas ao córrego também podem ocorrer nos períodos de maior pluviosidade, o que favorece o carreamento destes compostos até os corpos aquáticos, porque há evidências da presença de gado no entorno do P5 e do P4. Além da possibilidade deste fármaco presente na água ser consumido pelo gado que serve posteriormente de alimento para o ser humano. 


\section{REFERÊNCIAS}

AGENCIA NACIONAL DE VIGILANCIA SANITARIA- ANVISA. Resolução n 899, de 23 de maio de 2003. Disponível em: <http:www.anvisa.gov.br> Acesso: 03 jun. 2009.

BAUTITZ, I. R. Degradação de tetraciclina utilizando o processo foto-fenton. 2006. 85f. Dissertação (Mestrado em Química) - Instituto de Química, Universidade Estadual Paulista- UNESP, Araraquara, 2006.

BILA, D. M.; DEZOTTI, M. Desreguladores endócrinos no meio ambiente: efeitos e consequências. Química Nova, São Paulo, v.30, n.3, p.651-666, 2007.

GHISELLI, G.; JARDIM, W. Interferentes endócrinos no ambiente. Química Nova, São Paulo, v.30, n.3, p.695-706, 2007.

HALLING-SORENSEN, B.; NORS NIELSEN, S.; LANZKY, P. F.; INGERSLEV, F.; HOLTEN LUTZHOFT, H. C.; JORGENSEN, S. E. Occurrence, fate and effects of pharmaceutical substances in the environment- a review. Chemosphere, Oxford, v.36, n.2, p.357-393, 1998.

HENSCHEL, K. P., WENZEL, A., DIEDRICH, M.; FLIEDNER, A. Environmental hazard assessment of pharmaceuticals. Regulatory Toxicology and Pharmacology, New York, v.25, n.3, p.220-225, 1997.

INSTITUTO BRASILEIRO DE GEOGRAFIA E ESTATISTICA- IBGE. Cidades@: Três Lagoas MS. [S.I.: sn.], 2009. Disponível em:

<http://www.ibge.gov.br/cidadesat/default.php>. Acesso: 19 dez. 2009.

KASPRZYK-HORDERN, B. ; DINSDALE, R. M. ; GUWY, A. J. Multiresidue methods for the analysis of pharmaceuticals, personal care products and illicit drugs in surface water and wastewater by solid-phase extraction and ultra performance liquid chromatographyelectrospray tandem mass spectrometry. Analytical and Bioanalytical Chemistry, Heidelberg, v.391, n.4, p.1293-1308, 2008.

MOREIRA, M. A. L. 2006. As molduras vegetais do Córrego da Onça: Três Lagoas, Mato Grosso do Sul. 2006. 72f. Trabalho de Conclusão de Curso (Graduação em 
Geografia)- Departamento de Ciências Humanas, Universidade Federal de Mato Grosso do Sul- UFMS, Três Lagoas, 2006.

NEBOT, C., GIBB, S. W.; BOYD, K. G. Quantification of human pharmaceuticals in water samples by high performance liquid chromatography- tandem mass spectrometry. Analytica Chimica Acta, Amsterdam, v.598, n.1, p.87-94, 2007.

RAIMUNDO, C. C. C. Ocorrência de interferentes endócrinos e produtos farmacêuticos nas águas superficiais da bacia do rio Atibaia. 2007. 108f. Dissertação (Mestrado em Química) - Instituto de Química, Universidade Estadual de Campinas, Campinas, 2007.

RIBEIRO, F. A. L.; FERREIRA M. M. C. Planilha de validação: uma nova ferramenta para estimar figuras de mérito na validação de métodos analíticos univariados. Química Nova, São Paulo, v.31, n.1, p.164-171, 2008.

VULLIET, E.; CREN-OLIVE, C.; GRENIER-LOUSTALOT, M. F. Occurrence of pharmaceuticals and hormones in drinking treated from surface waters. Environmental Chemistry Letters, Berlim, v.7, n.1, p.1-12, 2009. 